\title{
Using Inhomogeneous Planar Layers as Impedance Matchers between Two Different Mediums
}

\author{
Mohammad Khalaj-Amirhosseini \\ Electrical Engineering Department, Iran University of Science and Technology, Narmak, 16846 Tehran, Iran \\ Correspondence should be addressed to Mohammad Khalaj-Amirhosseini, khalaja@iust.ac.ir
}

Received 8 October 2007; Accepted 28 January 2008

Recommended by Kamya Yekeh Yazdandoost

\begin{abstract}
A method is proposed to synthesize optimally inhomogeneous planar layers (IPLs) as impedance matchers between two mediums. First, the electric permittivity function of the IPL is expanded in a truncated Fourier series. Then, the optimum values of the coefficients of the series are obtained through an optimization approach. The usefulness of the proposed method is verified using some examples.
\end{abstract}

Copyright (C) 2008 Mohammad Khalaj-Amirhosseini. This is an open access article distributed under the Creative Commons Attribution License, which permits unrestricted use, distribution, and reproduction in any medium, provided the original work is properly cited.

\section{Introduction}

Impedance matching is a very important concept in microwave and antenna engineering. For example, in ground penetrating radars (GPRs), we need matching between the air and the soil (the earth) [1,2]. Usually the impedance matching is made into the structure of antennas. On the other hand, inhomogeneous planar layers (IPLs) are widely used in microwave and antenna engineering [3-5]. In this paper, we propose utilizing IPLs as impedance matchers between two mediums and introduce a method to synthesize them optimally in a desired frequency range. In fact, the IPLs are located between a free space designed antenna and the earth or walls. To optimally design IPLs, the electric permittivity function of them is expanded in a truncated Fourier series, first. Then, the optimum values of the coefficients of the series are obtained through an optimization approach. The usefulness of the proposed method is verified using some examples.

\section{Analysis of IPLs}

In this section, the frequency domain equations of the IPLs are reviewed. Figure 1 shows a typical IPL with thickness $d$, whose left medium is the free space and whose right (target) medium has the conductivity and electric permittivity $\sigma_{2}$ and $\varepsilon_{r 2}$, respectively. Also, the electric permittivity and the magnetic permeability functions of IPL are $\varepsilon_{r}(z)$ and 1 (for easy implementation). One way to fabricate the IPLs is to place several thin homogeneous dielectric layers beside each other. We are interested to utilize IPLs as impedance matchers between their right and left mediums, that is, between the target medium and free space. Two different polarizations are possible, one is the TM and the other is the TE. It is assumed that the incident plane wave propagates obliquely towards positive $x$ and $z$ directions with an angle of incidence $\theta_{i}$ and electric filed strength $E^{i}$. Of course, we know that the wave radiated by an antenna can be decomposed to many plane waves.

The differential equations describing IPLs have nonconstant coefficients and so except for a few special cases no analytical solution exists for them. There are some methods to analyze the IPLs such as finite difference [6], Taylor's series expansion [7], Fourier series expansion [8], the equivalent sources method [9], and the method of moments [10]. Of course, the most straightforward method is subdividing IPLs into $K$ uniform electrically short segments with length

$$
\Delta z=\frac{d}{K} \ll \lambda_{\min } \cong \frac{c}{f_{\max } \sqrt{\varepsilon_{r 2}}},
$$

in which $c$ is the velocity of the light and $f_{\max }$ is the maximum frequency of the analysis. The $A B C D$ parameters [11] of the 


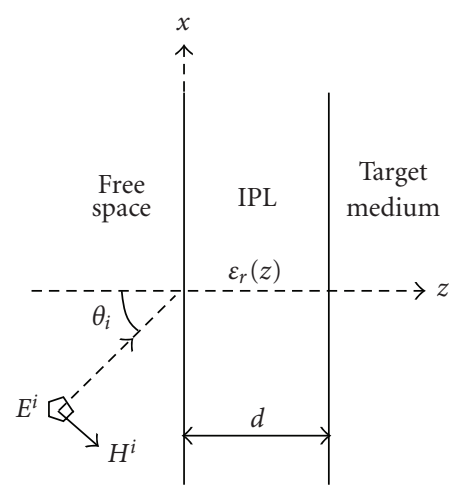

(a)

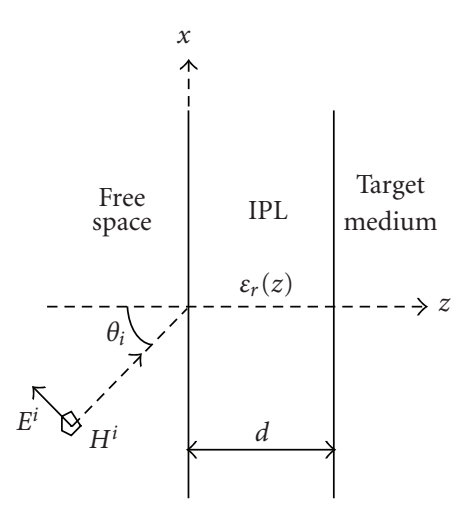

(b)

Figure 1: A typical IPL as an impedance matcher between two mediums: (a) TE polarization mode; (b) TM polarization mode.

IPL are obtained from those of the segments as follows:

$$
\left[\begin{array}{cc}
A & B \\
C & D
\end{array}\right]=\left[\begin{array}{cc}
A_{1} & B_{1} \\
C_{1} & D_{1}
\end{array}\right] \cdots\left[\begin{array}{cc}
A_{k} & B_{k} \\
C_{k} & D_{k}
\end{array}\right] \cdots\left[\begin{array}{cc}
A_{K} & B_{K} \\
C_{K} & D_{K}
\end{array}\right]
$$

where the $A B C D$ parameters of the $k$ th segment are as follows:

$$
\begin{aligned}
A_{k} & =D_{k}=\cos \left(\Delta \theta_{k}\right), \\
B_{k} & =Z_{c}^{2}\left((k-0.5) \Delta z, \theta_{i}\right) C_{k} j Z_{c}\left((k-0.5) \Delta z, \theta_{i}\right) \sin \left(\Delta \theta_{k}\right) .
\end{aligned}
$$

In (4) and (5),

$$
\Delta \theta_{k}=\frac{2 \pi f}{c} \sqrt{\varepsilon_{r}((k-0.5) \Delta z)-\sin ^{2}\left(\theta_{i}\right)} \Delta z
$$

is the electrical length of the $k$ th segment and $Z_{c}\left(z, \theta_{i}\right)$ is the characteristic impedance of the IPL, defined as the ratio of the transverse electric field to the transverse magnetic field given by

$$
Z_{c}\left(z, \theta_{i}\right)=\left\{\begin{array}{l}
\sqrt{\frac{\mu_{0}}{\varepsilon_{0}}} \frac{1}{\sqrt{\varepsilon_{r}(z)-\sin ^{2}\left(\theta_{i}\right)}}, \\
\sqrt{\frac{\mu_{0}}{\varepsilon_{0}}} \frac{1}{\varepsilon_{r}(z)} \sqrt{\varepsilon_{r}(z)-\sin ^{2}\left(\theta_{i}\right)},
\end{array}\right.
$$

TE,
Finally, the input impedance and reflection coefficient of the matcher are determined as follows:

$$
\begin{aligned}
Z_{\text {in }}\left(f, \theta_{i}\right) & =\frac{A Z_{L}\left(f, \theta_{i}\right)+B}{C Z_{L}\left(f, \theta_{i}\right)+D}, \\
\Gamma_{\text {in }}\left(f, \theta_{i}\right) & =\frac{Z_{\text {in }}\left(f, \theta_{i}\right)-Z_{S}\left(\theta_{i}\right)}{Z_{\text {in }}\left(f, \theta_{i}\right)+Z_{S}\left(\theta_{i}\right)},
\end{aligned}
$$

where

$$
\begin{aligned}
& Z_{L}\left(f, \theta_{i}\right)= \begin{cases}\sqrt{\frac{j \omega \mu_{0}}{\sigma_{2}+j \omega \varepsilon_{0} \varepsilon_{r 2}}} \frac{1}{\cos \left(\theta_{i}\right)}, & \text { TE, } \\
\sqrt{\frac{j \omega \mu_{0}}{\sigma_{2}+j \omega \varepsilon_{0} \varepsilon_{r 2}}} \cos \left(\theta_{i}\right), & \text { TM, }\end{cases} \\
& Z_{S}\left(\theta_{i}\right)= \begin{cases}\sqrt{\frac{\mu_{0}}{\varepsilon_{0}} \frac{1}{\cos \left(\theta_{i}\right)},} & \text { TE, } \\
\sqrt{\frac{\mu_{0}}{\varepsilon_{0}} \cos \left(\theta_{i}\right),} & \mathrm{TM},\end{cases}
\end{aligned}
$$

are the equivalent load and source impedances, respectively.

\section{Synthesis of IPLs}

In this section, a general method is proposed to optimally design the IPLs as impedance matchers. First, we consider the following truncated Fourier series expansion for the electric permittivity function

$$
\begin{aligned}
& \ln \left(\varepsilon_{r}(z)-1\right) \\
& =C_{0}+\sum_{n=1}^{N}\left(C_{n} \cos \left(\frac{2 \pi n z}{d}\right)+S_{n} \sin \left(\frac{2 \pi n z}{d}\right)\right)+\frac{E z}{d} .
\end{aligned}
$$

The latter term has been considered to prepare the possibility of having unequal values at $z=0$ and $z=d$. Also, the reason to use logarithm function at the left of (9) is to keep $\varepsilon_{r}(z) \geq$ 1. Moreover, utilizing truncated Fourier series expansion (9) does not create any discontinuity in the resultant IPL. An optimum-designed matcher has to have the input reflection coefficient as small as possible in a desired frequency range. Therefore, the optimum values of the coefficients $C_{n}, S_{n}$, and $E$ in (9) can be obtained through minimizing the following error function corresponding to $M$ frequencies $f_{1}<f_{2}<$ $\cdots<f_{M}$ inside the desired matching bandwidth and at zero angle of incidence.

$$
\text { Error }=\sqrt{\frac{1}{M} \sum_{m=1}^{M}\left|\Gamma_{\text {in }}\left(f_{m}, 0\right)\right|^{2}} .
$$

Moreover, the defined error function should be restricted by some constraints such as having a minimum matching at the largest desired angle of incidence, $\theta_{i, \max }$, as the following:

$$
\left|\Gamma_{\text {in }}\left(f_{m}, \theta_{i, \max }\right)\right| \leq \rho_{\max }, \quad \forall m=1,2, \ldots, M .
$$


TABLE 1: The unknown coefficients of the truncated Fourier series related to the synthesized IPLs with $d=10 \mathrm{~cm}$.

\begin{tabular}{lccccccccccccc}
\hline & $C_{0}$ & $C_{1}$ & $C_{2}$ & $C_{3}$ & $C_{4}$ & $C_{5}$ & $S_{1}$ & $S_{2}$ & $S_{3}$ & $S_{4}$ & $S_{5}$ & $E$ \\
\hline Dry/TE & -2.2846 & -0.4614 & -0.0279 & 0.2642 & 0.7325 & 0.3623 & -1.4137 & -0.9213 & -0.7610 & -0.4006 & 0.4135 & 2.0057 \\
Dry/TM & -2.3059 & -0.5146 & -0.0564 & 0.2213 & 0.7082 & 0.4512 & -1.4236 & -0.9258 & -0.7737 & -0.4923 & 0.4174 & 2.0643 \\
Moist/TE & -3.0211 & -0.1310 & 0.0685 & 0.1639 & 1.0853 & 0.5526 & -1.7717 & -1.0275 & -0.9858 & -0.8211 & 0.7896 & 2.8266 \\
Moist/TM & -5.0000 & 0.1260 & 0.0578 & -0.7545 & 0.1564 & 1.8839 & -2.2487 & -0.5031 & -0.5168 & -2.4156 & 0.6678 & 4.4385 \\
\hline
\end{tabular}

TABLE 2: The unknown coefficients of the truncated Fourier series related to the synthesized IPLs with $d=7 \mathrm{~cm}$.

\begin{tabular}{cccccccccccccc}
\hline & $C_{0}$ & $C_{1}$ & $C_{2}$ & $C_{3}$ & $C_{4}$ & $C_{5}$ & $S_{1}$ & $S_{2}$ & $S_{3}$ & $S_{4}$ & $S_{5}$ & $E$ \\
\hline Dry/TE & -1.6962 & -0.8780 & 0.2844 & 1.9003 & 0.3355 & 0.6701 & -2.5874 & -2.2971 & -1.0016 & 0.5989 & 0.4548 & 0.0789 \\
Dry/TM & -1.5613 & -0.3715 & 0.2027 & 1.3688 & 1.1148 & -0.4685 & -2.9285 & -1.6279 & -1.3631 & 1.2224 & -0.4454 & -0.3434 \\
\hline
\end{tabular}

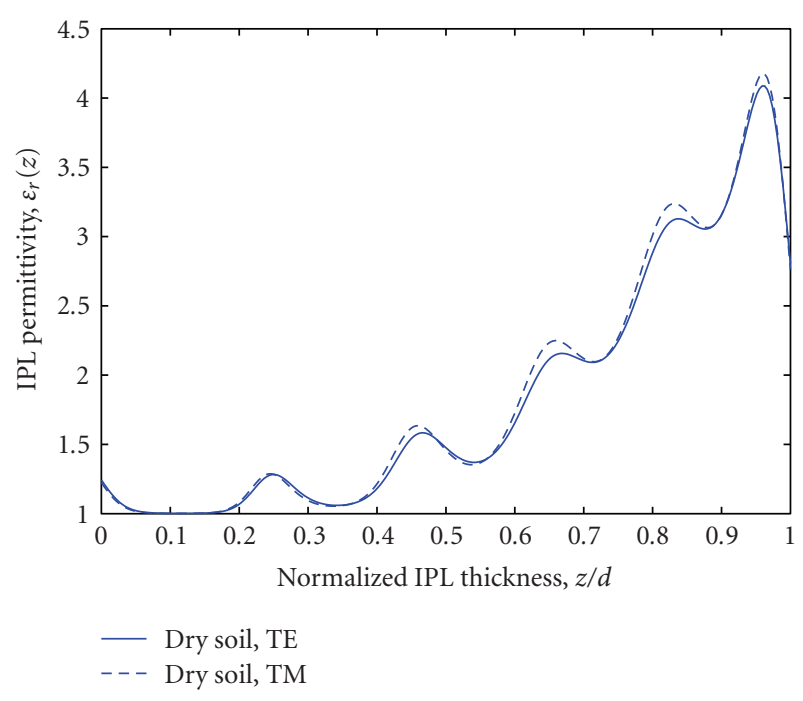

Figure 2: The electric permittivity function $\varepsilon_{r}(z)$ to match to the dry soil with $d=10 \mathrm{~cm}$.

It is noticeable that in contrary to condition (11), the error function (10) is independent of the wave polarization because TE and TM polarizations are identical for the zero angle of incidence. Also, one can consider the following truncated Fourier series expansion for the electric permittivity function, instead of (9),

$$
\ln \left(\varepsilon_{r}(z)-1\right)=\sum_{n=0}^{N} C_{n}^{\prime} \cos \left(\frac{\pi n z}{d}\right) .
$$

\section{Examples and Results}

We would like to design an IPL with thickness $d=10 \mathrm{~cm}$ (a practical and feasible chosen) as an impedance matcher in a frequency range 2.0 to $4.0 \mathrm{GHz}$ (an octave bandwidth), considering $\theta_{i, \max }=60^{\circ}$ and $\rho_{\max }=0.316=-10 \mathrm{~dB}$. We consider two cases for the target medium; dry soil $\left(\varepsilon_{r 2}=4\right.$ and $\left.\sigma_{2}=0.001\right)$ and moist soil $\left(\varepsilon_{r 2}=10\right.$ and $\left.\sigma_{2}=0.01\right)$. Using the proposed optimization approach, considering

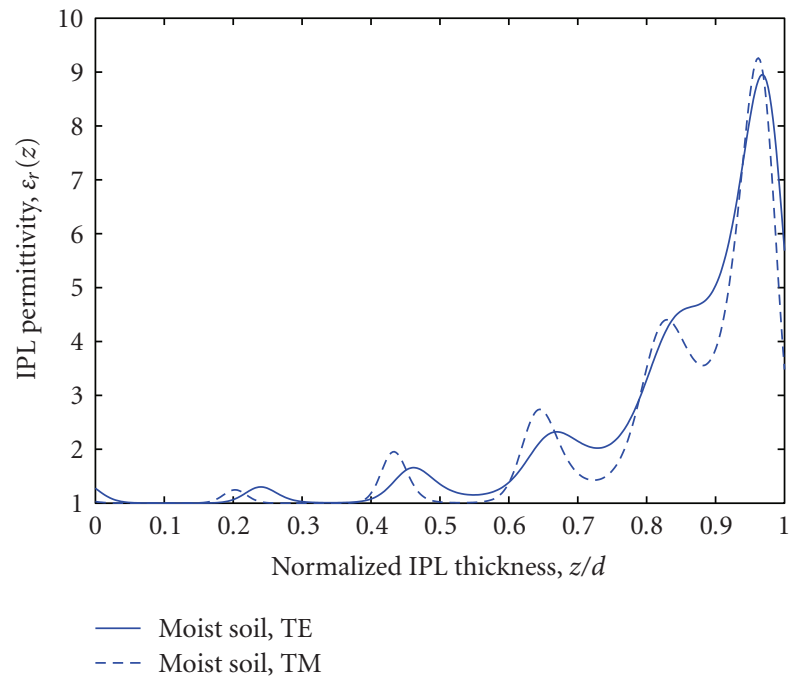

FIgure 3: The electric permittivity function $\varepsilon_{r}(z)$ to match to the moist soil with $d=10 \mathrm{~cm}$.

$N=5$ spatial harmonics and $M=21$ frequencies with equal distance of $100 \mathrm{MHz}$, four IPLs were synthesized. The unknown coefficients of the truncated Fourier series related to the synthesized IPLs are written in Table 1. Figures 2 and 3 illustrate the electric permittivity function $\varepsilon_{r}(z)$ to match to either the dry or moist soils, respectively, assuming TE or TM polarizations. Figures 4 to 7 illustrate the magnitude of the input reflection coefficient $\left|\Gamma_{\text {in }}(f)\right|$ corresponding to the dry or moist soils illuminated by TE or TM polarizations waves. It is observed that the designed IPLs yield a good impedance matching in the desired frequency band and angular range. Meanwhile, the reflection coefficient degrades with increasing the angle of incidence. The variation of the permittivity of designed IPLs in TM mode is slightly more than that in TE mode. Also, the resulted matching efficiency for the moist soil is less than that for the dry soil. To show the effect of the thickness of IPLs, we reduce $d$ from $10 \mathrm{~cm}$ to $7 \mathrm{~cm}$. The unknown coefficients of the truncated Fourier series, the electric permittivity function $\varepsilon_{r}(z)$ and the magnitude of the input reflection coefficient $\left|\Gamma_{\text {in }}(f)\right|$ 


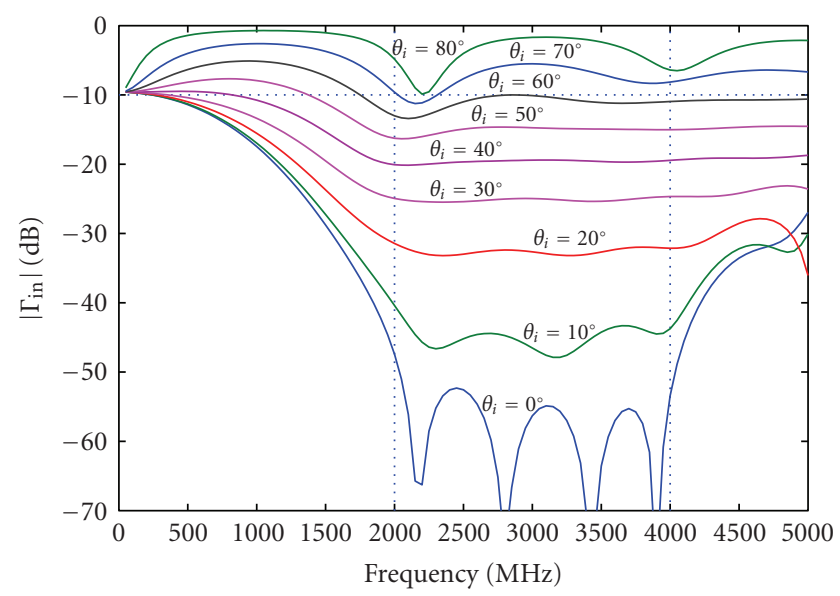

Figure 4: The magnitude of $\left|\Gamma_{\text {in }}(f)\right|$ corresponding to the dry soil, $d=10 \mathrm{~cm}$ and TE polarization.

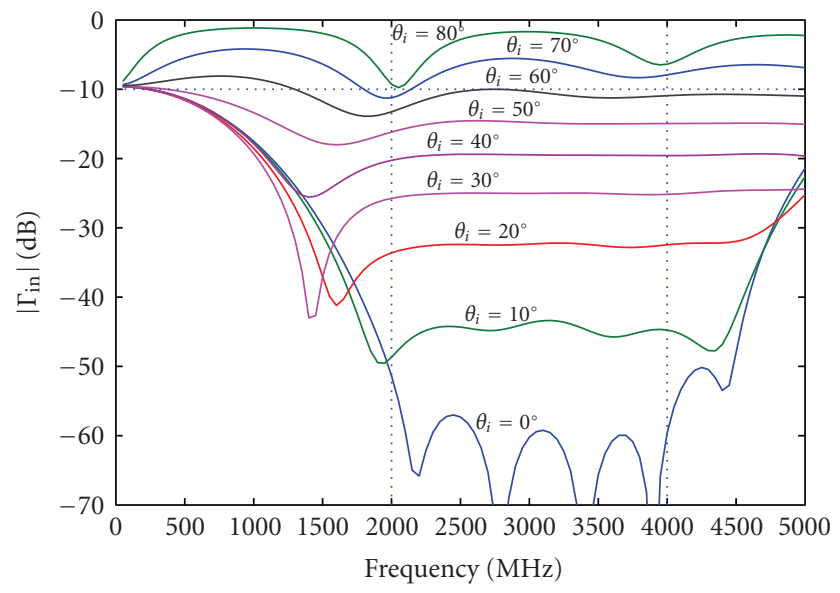

FIgURE 5: The magnitude of $\left|\Gamma_{\text {in }}(f)\right|$ corresponding to the dry soil, $d=10 \mathrm{~cm}$ and TM polarization.

corresponding to the dry soil with $d=7 \mathrm{~cm}$ are shown in Table 2 and Figures 8-10, respectively. Comparing Figures 9, 10 with Figures 4, 5 results that as the thickness of the IPL is chosen larger, its matching efficiency can be increased. Also, comparing Figure 8 with Figure 2 results that as the thickness of the IPL is chosen smaller, the necessary variations of the permittivity function is increased and consequently its implementation becomes more difficult.

\section{Conclusion}

Inhomogeneous planar layers (IPLs) were utilized as impedance matchers between two mediums and a method was introduced to optimally synthesize them in a desired frequency band. First, the electric permittivity function of the IPL is expanded in a truncated Fourier series. Then, the optimum values of the coefficients of the series are obtained through an optimization approach. The usefulness

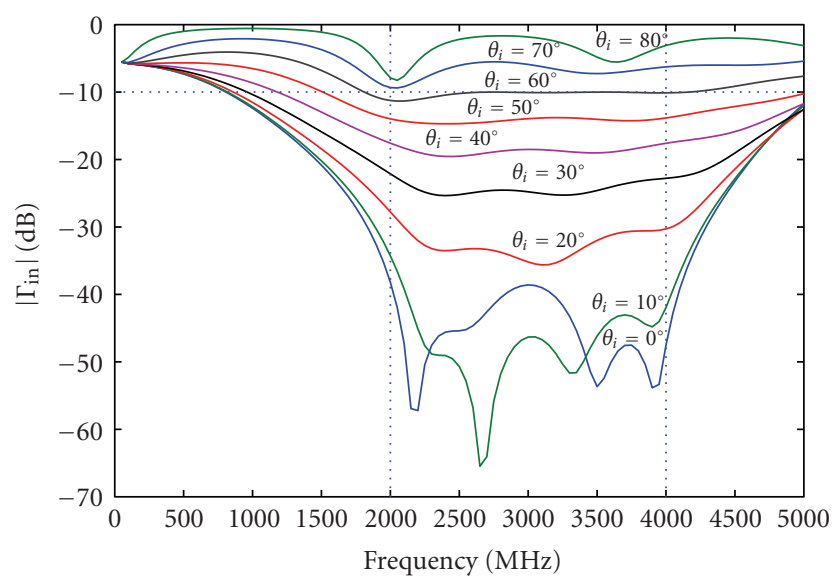

FIgURE 6: The magnitude of $\left|\Gamma_{\text {in }}(f)\right|$ corresponding to the moist soil, $d=10 \mathrm{~cm}$ and TE polarization.

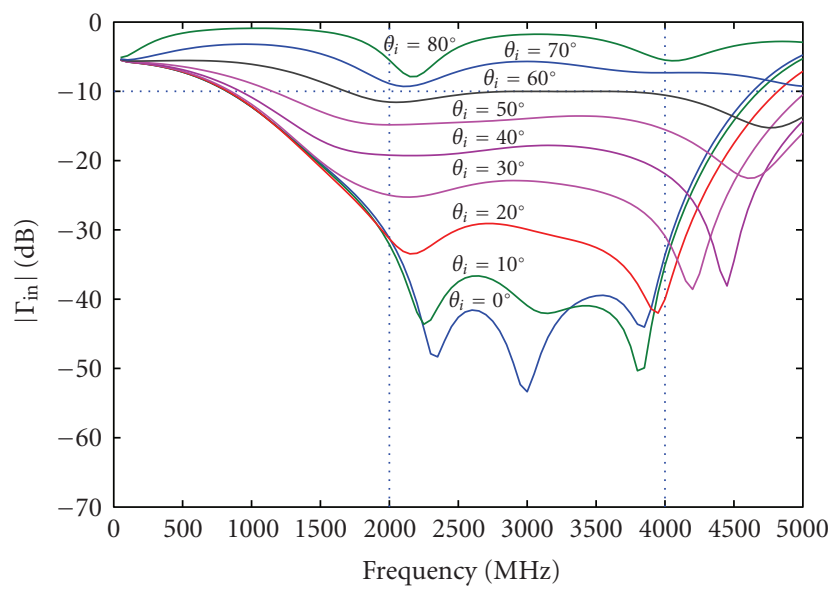

Figure 7: The magnitude of $\left|\Gamma_{\text {in }}(f)\right|$ corresponding to the moist soil, $d=10 \mathrm{~cm}$ and TM polarization.

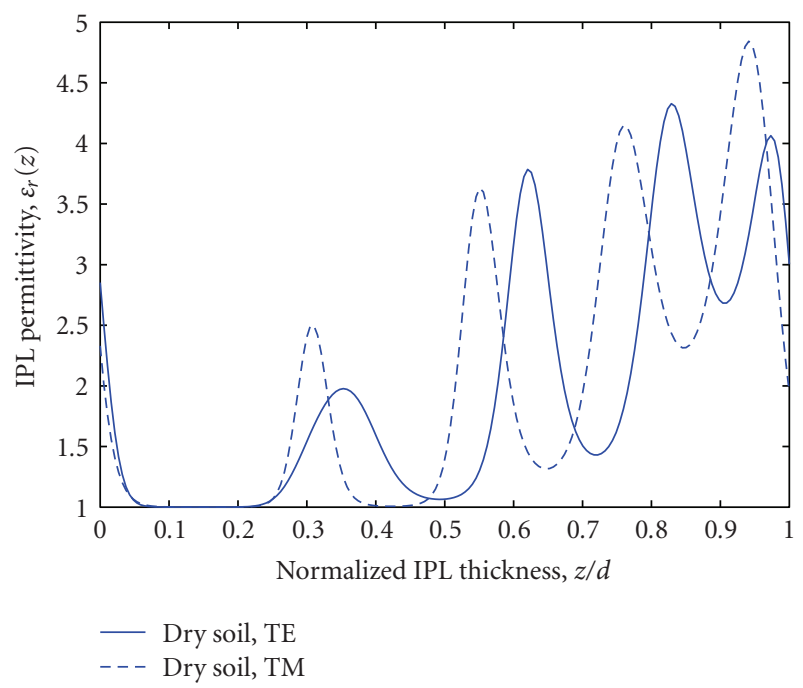

Figure 8: The electric permittivity function $\varepsilon_{r}(z)$ to match to the dry soil with $d=7 \mathrm{~cm}$. 


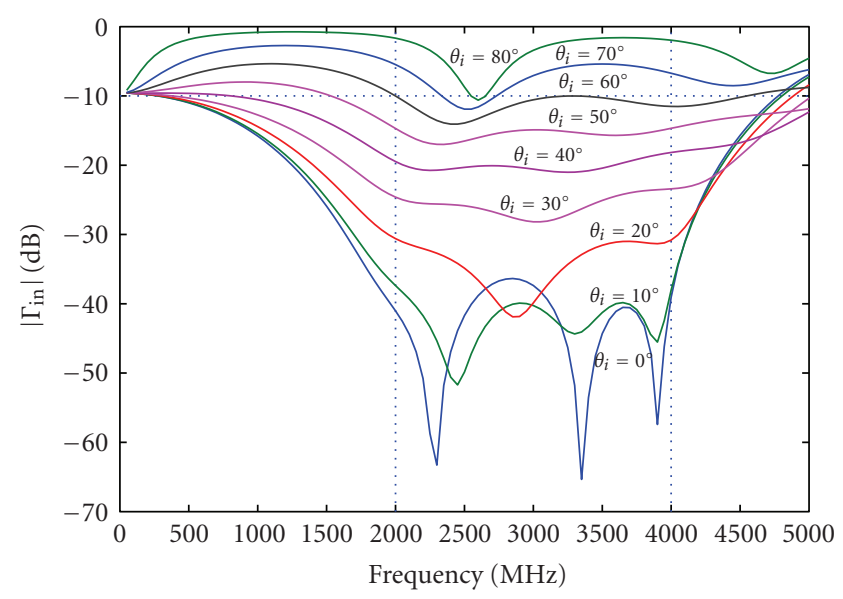

Figure 9: The magnitude of $\left|\Gamma_{\text {in }}(f)\right|$ corresponding to the dry soil, $d=7 \mathrm{~cm}$ and TE polarization.

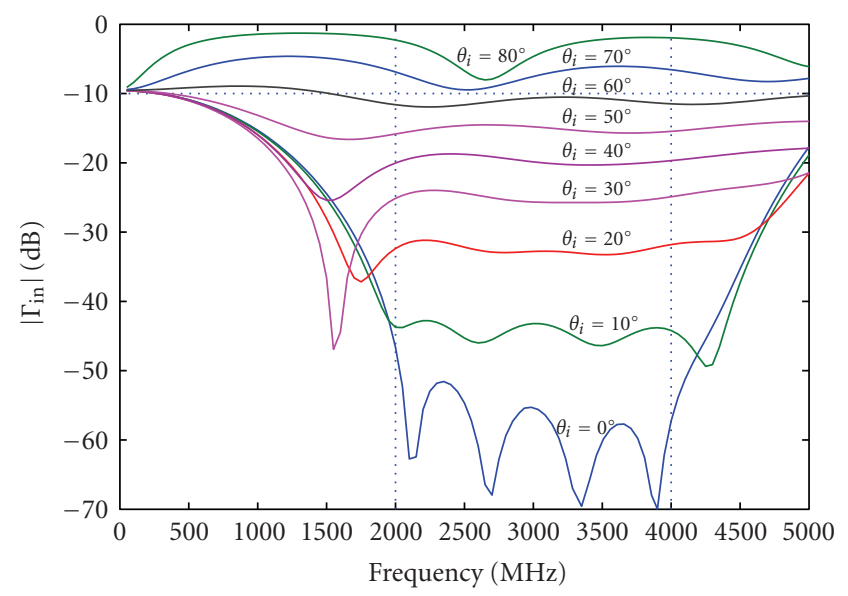

FIgURE 10: The magnitude of $\left|\Gamma_{\text {in }}(f)\right|$ corresponding to the dry soil, $d=7 \mathrm{~cm}$ and TM polarization.

of the proposed method is verified using some examples. It is observed that the solutions yield a good impedance matching and thicker matching layer may have higher efficiency and easier fabrication. The proposed method can be extended for IPLs, whose magnetic permeability is inhomogeneous solely or along with their electric permittivity. Also, we can consider IPLs for spherical wave fronts instead of planar ones in the future.

\section{References}

[1] J. S. Mellett, "Ground penetrating radar applications in engineering, environmental management, and geology," Journal of Applied Geophysics, vol. 33, no. 1-3, pp. 157-166, 1995.

[2] E. D. Guy, J. J. Daniels, S. J. Radzevicius, and M. A. Vendl, "Demonstration of using crossed dipole GPR antennae for site characterization," Geophysical Research Letters, vol. 26, no. 22, pp. 3421-3424, 1999.

[3] F. Bilotti, A. Toscano, and L. Vegni, "Very fast design formulas for microwave nonhomogeneous media filters," Microwave and Optical Technology Letters, vol. 22, no. 3, pp. 218-221, 1999.

[4] L. Vegni and A. Toscano, "Full-wave analysis of planar stratified media with inhomogeneous layers," IEEE Transactions on Antennas and Propagation, vol. 48, no. 4, pp. 631-633, 2000.

[5] A. Toscano, L. Vegni, and F. Bilotti, "A new efficient method of analysis for inhomogeneous media shields and filters," IEEE Transactions on Electromagnetic Compatibility, vol. 43, no. 3, pp. 394-399, 2001.

[6] M. Khalaj-Amirhosseini, "Analysis of lossy inhomogeneous planar layers using finite difference method," Progress in Electromagnetics Research, vol. 59, pp. 187-198, 2006.

[7] M. Khalaj-Amirhosseini, "Analysis of lossy inhomogeneous planar layers using Taylor's series expansion," IEEE Transactions on Antennas and Propagation, vol. 54, no. 1, pp. 130-135, 2006.

[8] M. Khalaj-Amirhosseini, "Analysis of lossy inhomogeneous planar layers using fourier series expansion," IEEE Transactions on Antennas and Propagation, vol. 55, no. 2, pp. 489-493, 2007.

[9] M. Khalaj-Amirhosseini, "Analysis of lossy inhomogeneous planar layers using equivalent sources method," Progress in Electromagnetics Research, vol. 72, pp. 61-73, 2007.

[10] M. Khalaj-Amirhosseini, "Analysis of lossy inhomogeneous planar layers using the method of moments," Journal of Electromagnetic Waves and Applications, vol. 21, no. 14, pp. 1925-1937, 2007.

[11] R. E. Collin, Foundations for Microwave Engineering, McGrawHill, New York, NY, USA, 1996. 

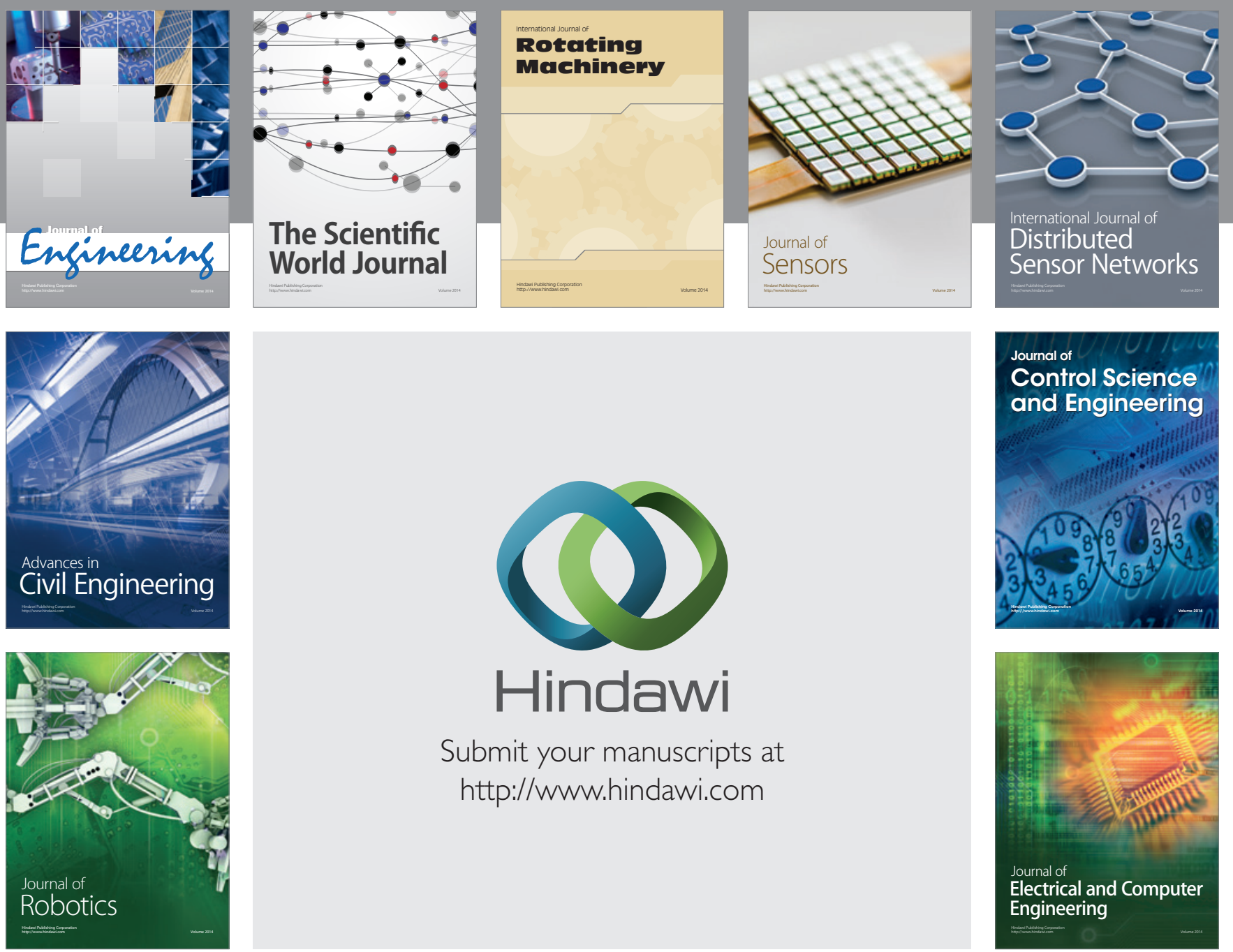

Submit your manuscripts at

http://www.hindawi.com
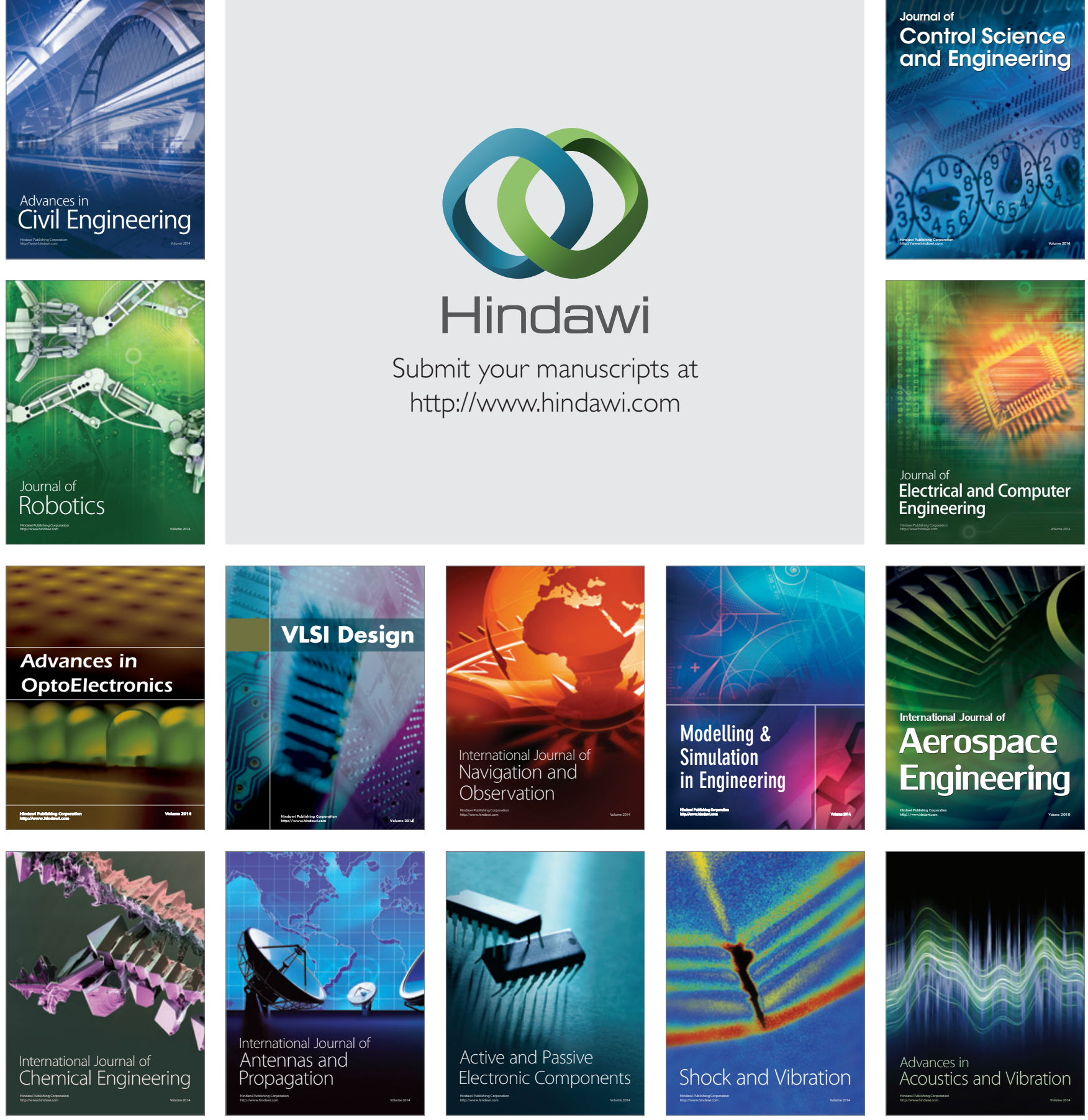